\title{
Effects of Placement, Attachment, and Weight Classification on Pedometer Accuracy
}

\author{
Susan V. Graser \\ William J. Vincent \\ bill_vincent@byu.edu \\ Robert P. Pangrazi
}

Follow this and additional works at: https://scholarsarchive.byu.edu/facpub

Part of the Exercise Science Commons

\section{Original Publication Citation}

Effects of Placement, Attachment, and Weight Classification on Pedometer Accuracy. Journal of Physical Activity and Health, Vol. 4, No. 4, pp.359 - 369, October 27. S. Vincent Graser, R. P.

Pangrazi, \& W. J. Vincent.

\section{BYU ScholarsArchive Citation}

Graser, Susan V.; Vincent, William J.; and Pangrazi, Robert P., "Effects of Placement, Attachment, and Weight Classification on Pedometer Accuracy" (2007). Faculty Publications. 231.

https://scholarsarchive.byu.edu/facpub/231 


\title{
Effects of Placement, Attachment, and Weight Classification on Pedometer Accuracy
}

\author{
Susan Vincent Graser, Robert P. Pangrazi, \\ and William J. Vincent
}

\begin{abstract}
Background: The purpose was to determine if waist placement of the pedometer affected accuracy in normal, overweight, and obese children, when attaching the pedometer to the waistband or a belt. Methods: Seventy-seven children (ages 10-12 years) wore 5 pedometers on the waistband of their pants and a belt at the following placements: navel (NV), anterior midline of the right thigh (AMT), right side (RS), posterior midline of the right thigh (PMT), and middle of the back (MB). Participants walked 100 steps on a treadmill at $80 \mathrm{~m} \cdot \mathrm{min}^{-1}$. Results: The RS, PMT, and MB sites on the waistband and the AMT and RS sites on the belt produced the least error. Conclusions: Of these sites, the RS placement is recommended because of the ease of reading the pedometer during activity. Using a belt did not significantly improve accuracy except for normal weight groups at the NV placement site.
\end{abstract}

Key Words: physical activity, measurement, youth

Pedometers are a popular tool for monitoring physical activity. Previous research has validated the use of the pedometer as an appropriate tool for monitoring physical activity. ${ }^{1-4}$ Pedometers are a popular tool for monitoring physical activity in physical education and free-living settings, and recommendations for such use are available. ${ }^{5}$ In addition, pedometers are cost efficient as compared to accelerometers or heart rate monitors.

There are limitations to using the pedometer for physical activity research. A shortcoming, particularly for researchers, is the pedometer's inability to measure intensity. Pedometers measure the cumulative number of steps an individual takes but are unable to indicate the intensity level of those steps. Another concern for researchers is the integrity of the data collected. When gathering pedometer data, there is always the risk of participants tampering with the pedometer (eg, shaking it to give the illusion of more steps, accidentally hitting the reset button and losing data) thus compromising the integrity of the data. Some researchers use sealed pedometers to help prevent tampering. ${ }^{6,7}$

Vincent Graser is with Brigham Young University, 249G SFH, Provo, UT; Pangrazi is professor emeritus at Arizona State University; and Vincent is with Brigham Young University, Provo, UT. 
A strong need exists for a pedometer protocol to assure accurate measurements among participants of various weight classifications (normal, overweight, and obese). A spring-loaded pedometer must be in the vertical plane to function correctly. If the pedometer is tilted, it may be inaccurate or not count steps at all. The piezoelectric pedometer uses a horizontal cantilevered beam with a weight on the end. The beam compresses a piezoelectric crystal when acceleration occurs to record steps. The piezoelectric pedometers appear to be less affected by tilt when compared to spring-lever pedometers. ${ }^{8}$ Typically, the piezoelectric pedometers cost more than spring-lever pedometers.

Most manufacturers recommend the pedometer be placed on the waistband of a person's pants or on a belt positioned directly above the midline of the thigh (on either the right or left side). This usually places the pedometer in a vertical plane. Most research to this point has followed this recommendation. ${ }^{1,9,10}$ However, this positioning is often problematic for individuals who are overweight or have excessive abdominal fat. ${ }^{11}$ Abdominal fat may cause the pedometer to tip forward and not be in a vertical position causing the pedometer to count inaccurately. ${ }^{12}$

Several studies have attempted to evaluate whether the placement of the pedometer made a difference in accuracy. Shepherd et al. ${ }^{11}$ conducted a study in which adult participants engaged in 4 activities (400-m walk, stair descent, 10-m walk, and stair ascent) while wearing a pedometer at the waistline near the anterior superior iliac spine. Participants walked 10 steps and then the placement was adjusted for improved accuracy, if needed. In participants with a body mass index (BMI) less than 30, the pedometer averaged $1.6 \%$ error. However, in participants with a BMI greater than 30, the pedometer averaged $6.1 \%$ error. Additionally, there was a positive linear correlation between absolute error and BMI $(r=.792)$.

Swartz et al. ${ }^{13}$ compared pedometer accuracy based on placement in overweight adults with the pedometer at the anterior midline of the thigh, midaxillary line, and the posterior midline of the thigh. Participants walked on a treadmill at 5 walking speeds $\left(54,67,80,94,107 \mathrm{~m} \cdot \mathrm{min}^{-1}\right)$ for 3 minutes at each speed while wearing the 3 pedometers. A researcher counted by hand the number of steps each participant took to check for accuracy. Participants were grouped by BMI as normal weight $(<25)$, overweight (25-29.9) and obese (>30). Placement of the pedometer made no significant difference in the accuracy of data for any of the groups.

Jago et al. ${ }^{14}$ determined that pedometer location made no difference in accuracy of step counts in boys ages 11 to 15 years. Three pedometers were worn (on the right hip, center (navel), and left hip) on an elastic belt while participants walked on a hard surface at 3 different speeds. Results concluded that there was no difference in accuracy when the pedometer was placed at the 3 different locations. Differences in accuracy were most affected by stature, not pedometer location or adiposity.

When spring-lever pedometers were compared with piezoelectric pedometers, it was determined that tilt was the most important factor affecting accuracy of the spring-lever pedometer. ${ }^{8}$ The piezoelectric pedometer was not affected by tilt in adult participants who were overweight or obese.

To date, the effect of excess body fat on the accuracy of the pedometer has not been established with younger children using a spring-lever pedometer. Therefore, 
the purposes of this study were to (1) determine if different waist placement sites of a spring-levered pedometer affect the accuracy of step counts in normal weight, overweight, and obese children; (2) evaluate if placement of the pedometer on a belt compared to the waistband of a participant's clothing impacted accuracy; and (3) evaluate if the type of pant worn affected accuracy.

\section{Methods}

\section{Participants}

Seventy-seven children (ages 10-12 years), from an elementary school in a southwest state, volunteered to participate. All children returned an informed assent and consent form signed by themselves and their parents. Approval was obtained from the school's principal and the University's Institutional Review Board, which regulates research with human subjects.

\section{Instruments}

The Walk4Life LS 2505 pedometer, which measures steps and activity time, was used in this study. Research has shown that pedometers are valid and reliable instruments for measuring ambulatory physical activity. ${ }^{1,3,4,9,15}$ Recent research found the Walk4Life pedometer to be accurate within $\pm 1 \%$ error at a speed of $80 \mathrm{~m} \cdot \mathrm{min}^{-1}$ or greater. ${ }^{2}$ Beets et al. ${ }^{3}$ also found the Walk4Life pedometer to be accurate when compared to hand-counted steps (ICC $\geq 0.985$ ). Both Crouter et al. ${ }^{2}$ and Beets et al. ${ }^{3}$ identified the Walk4Life pedometer to be an appropriate measurement tool to be used in laboratory or field studies.

A Sole TT8 motorized treadmill was set up in a secure location at the school. The treadmill was calibrated on site using standard protocols ${ }^{10}$ and was found to be accurate to within $\pm 1 \%$ error.

\section{Procedures}

\section{The Shake Test}

Prior to use in the study, 6 pedometers were tested using a standardized shake test. ${ }^{16}$ The shake test ensured the accuracy of the pedometers in a lab setting prior to use in the study. Each pedometer was placed vertically in a cell of the shake test box, and the researcher shook the box 100 times. One end of the box maintained contact with the table in order to minimize extraneous movements while the other end of the box was moved in the vertical direction 100 times. Counts from each pedometer were recorded. This protocol has been validated in previous research. ${ }^{16}$ All 6 pedometers were found to be accurate within $\pm 1 \%$ error. At the conclusion of the study, the same pedometers were tested again to determine that they were still measuring steps accurately. Five out of six of the pedometers were accurate within $\pm 1 \%$ error and one pedometer was accurate within $\pm 2 \%$ error at the conclusion of the study. 


\section{Anthropometric Measures}

With shoes off and any coats or extra clothing removed, participants were measured for height, weight, and abdominal circumference. A stadiometer was used to measure height and a Tanita Body composition Analyzer BF-350 was used to measure weight. BMI was calculated as weight in kilograms divided by height in meters squared. Abdominal circumference was measured, at the level of the navel, using a tension gauge measuring tape through one layer of clothing (shirt, undershirt). Abdominal circumference was measured twice to the nearest $0.1 \mathrm{~cm}$, and the average of the two measurements was recorded for data analysis. ${ }^{17}$

\section{Pedometer Placement Walking Test}

Participants practiced walking and stepping on and off the moving treadmill prior to testing to assure they were comfortable with the protocol. After practicing walking on the treadmill, 5 pedometers were fastened to the waistband of participants' pants or to a belt located at the following placements: navel (NV), anterior midline of the right thigh (AMT), right side (midaxillary line) (RS), posterior midline of the right thigh (PMT), and middle of the back (MB). There were 2 phases to the walking test: (1) participants attached the pedometers directly to the waistband of their own clothing without a belt, and (2) participants attached the pedometers to a standardized belt. The researcher assisted the participant to be sure the pedometers were located in the correct place and were reset to zero before beginning each walking test.

The standardized belt, purchased from Walk4Life (Plainfield, Ill), is designed to be used in physical education classes for pedometer attachment. The belt measures approximately 2.5 in wide and is made of Velcro. The purpose of the belt is to ensure that the pedometer remains in the correct vertical position and standardize how the pedometer is anchored to the body.

Youth often wear different types of pants or shorts with various elastic waistbands that may be less secure and/or unstable. The type of pant worn by participants was recorded to determine if pant type affected the pedometer accuracy. Pants were classified as follows: Docker-type pant, Levi-type jeans, or sweat pants. No other type of pant was worn in this study.

The order of this 2-part walking test was reversed for each participant to control for any order effect. Both walking tests (waistband test, belt test) were administered with the same protocol.

Prior studies have determined that pedometers may be less accurate at slow speeds. Crouter et al. ${ }^{2}$ found $80 \mathrm{~m} \cdot \mathrm{min}^{-1}(3.0 \mathrm{mph})$ to be the minimal speed where accuracy was acceptable. After the pedometers were correctly placed, the participant straddled the treadmill belt by standing on the side rails, and the pedometers were reset to zero. The treadmill was set to a speed of $80 \mathrm{~m} \cdot \mathrm{min}^{-1}$. When the participant was ready, he or she stepped onto the treadmill and walked for 100 steps. The researcher counted the number of actual steps using a hand counter. When the participant reached 95 steps, the researcher counted the last 5 steps out loud so the participant would know when to step off the treadmill onto the side rails. Participants were instructed to take their 100th step on the side rail. The researcher then read and recorded the step counts on each of the 5 pedometers. Each participant completed this process for the waistband test and the belt test. 
Pedometer accuracy was determined using 2 methods. First, error rates were determined for each placement site. This was purely descriptive in nature in an attempt to determine which pedometer sites produced the least and the most error. A criterion of $\pm 5 \%$ pedometer error was considered acceptable. This criterion was used to allow for pedometer error as well as some participant error in getting off the treadmill on exactly the 100th step. The participants' abilities to count the final 5 steps and step off the treadmill on exactly step number 100 varied. Researchers observed many participants who were able to step off the treadmill on exactly step 100 while others would step off too early or too late. Therefore, a criterion of $\pm 5 \%$ error allows for error on the part of the pedometer $(1 \%-2 \%$ based on the shake test) and the participants (2-3 missed or additional steps), yet it still represents an acceptable level of accuracy. Error greater than $\pm 5 \%$ was attributed to other factors. For example, excessive abdominal fat may cause the pedometer to move out of the vertical plane or cushion the ground force and hinder its ability to register movement. Placement of the pedometer on the waistband of clothing versus on a belt may also cause error depending on the stability of the placement.

Second, pedometer step counts were compared statistically across the placement sites and among the weight groups. Data points that were greater than 3 standard deviations from a mean of 100 steps were considered outliers and were removed from the analysis.

\section{Results}

Descriptive statistics for height, weight, abdominal circumference, and BMI were calculated and can be found in Table 1. To determine the effect of excess weight on the accuracy of the pedometer at various placements on the body, participants were classified as normal weight $(n=50)$, overweight $(n=15)$, or obese $(n=12)$ based on their BMI and referenced to international cut points. ${ }^{18}$ These international standards provide cut points based on a broad sample of children, including children from the United States. Using the international standards provides an opportunity to make comparisons of these results with a wide range of studies. The international cut points are classified by age and sex and are presented in Table 2.

Table 1 Means and Standard Deviations for Height, Weight, Abdominal Circumference, and Body Mass Index (BMI)

\begin{tabular}{lrrrc}
\hline & $\begin{array}{c}\text { Normal } \\
\text { weight } \\
\mathbf{n}=\mathbf{5 0}\end{array}$ & $\begin{array}{c}\text { Overweight } \\
\mathbf{n = 1 5}\end{array}$ & $\begin{array}{c}\text { Obese } \\
\mathbf{n = 1 2}\end{array}$ & $\begin{array}{c}\text { All } \\
\text { participants } \\
\mathbf{N}=\mathbf{7 7}\end{array}$ \\
\hline Age (y) & $10.96 \pm 0.76$ & $11.00 \pm 0.76$ & $11.33 \pm 0.78$ & $11.03 \pm 0.76$ \\
Height (m) & $147.19 \pm 9.12$ & $150.57 \pm 8.28$ & $155.40 \pm 6.65$ & $149.13 \pm 9.04$ \\
Weight (kg) & $38.97 \pm 7.25$ & $52.21 \pm 8.10$ & $68.57 \pm 11.82$ & $46.16 \pm 13.66$ \\
Abdominal & & & & \\
circumference (cm) & $66.12 \pm 4.88$ & $81.66 \pm 5.83$ & $91.14 \pm 7.77$ & $73.04 \pm 11.33$ \\
BMI & $17.85 \pm 1.66$ & $22.86 \pm 1.58$ & $28.20 \pm 2.84$ & $20.44 \pm 4.30$ \\
\hline
\end{tabular}




\section{Table 2 International Cut-Off Points for Body Mass Index for Overweight and Obesity for Boys and Girls Ages 10 to 12 Years}

\begin{tabular}{llllll}
\hline & \multicolumn{2}{c}{ BMl $25 \mathrm{~kg} / \mathbf{m}^{2}$} & & \multicolumn{2}{c}{ BMI $\mathbf{3 0} \mathbf{~ k g} / \mathbf{m}^{2}$} \\
\cline { 2 - 3 } \cline { 5 - 6 } Age & Boys & Girls & & Boys & Girls \\
\hline 10 & 19.84 & 19.86 & & 24.00 & 24.11 \\
11 & 20.55 & 20.74 & & 25.10 & 25.42 \\
12 & 21.22 & 21.68 & & 26.02 & 26.67 \\
\hline
\end{tabular}

Note: These values represent the BMIs on children that are equivalent to an adult BMI of 25 or 30 . Reprinted with permission from the BMJ Publishing Group; Cole TJ, Bellizzi MC, Flegal KM, Dietz WH. Establishing a standard definition for child overweight and obesity worldwide: international survey. BMJ. 2000;320:1-6.

\section{Descriptive View of Error Rates by Placement Sites}

The percentage of pedometers with error greater than $\pm 5 \%$ for each placement site, waistband or belt test, and weight classification can be found in Table 3. During the waistband test, the placement site which produced the least amount of error $(6.6 \%$, weighted mean) was the right side (RS) when all participants were combined. The navel (NV) placement site produced the most error (31\%) for all groups combined, making it the least desirable position.

During the belt test, the 2 sites with the least amount of error were AMT and RS. For normal weight, overweight, and obese participants combined, the AMT placement had an error rate of $2.6 \%$ and the RS site produced an error rate of $5.3 \%$. It should be noted from the descriptive perspective in Table 3, that the NV placement site had less error when using a belt compared to a waistband; however, it was still the placement site that produced the most error in each case.

\section{Statistical Comparisons on Placement Sites}

To determine if BMI classification had an effect on mean step counts at various waist placement sites, an ANOVA on pedometer-recorded step counts among the three weight groups (normal, overweight, and obese) was performed on each placement site during the waistband and the belt tests. To reduce the probability of making a type 1 error when conducting multiple ANOVAs, a Bonferroni adjustment to the alpha level was applied $(P=.05 / 5=.01)$ and statistical significance was declared if $P \leq .01$.

On the waistband test, no significant differences were found for the NV $(\mathrm{F}=0.670, P=.515), \mathrm{RS}(\mathrm{F}=0.364, P=.696), \mathrm{PMT}(\mathrm{F}=3.89, P=.025)$ or $\mathrm{MB}$ $(\mathrm{F}=0.670, P=.515)$ sites (Table 4$)$. This indicates that the obesity factor had no effect on these 4 sites; however, while differences at the NV site were not significant, all 3 weight groups had step counts that were greater than $\pm 5 \%$ error based on 100 steps. A significant difference was found among the weight classification groups at the AMT placement site $(\mathrm{F}=8.610, P<.001)$. Tukey's post hoc test revealed significant differences at the AMT site between the obese group and both the overweight and normal groups (Table 4). 
Table 3 Percent of Pedometers With Greater Than $\pm 5 \%$ Error by Placement Site, Waistband or Belt Test, and Weight Classification

\begin{tabular}{lrrrrr}
\hline & NV & AMT & RS & PMT & MB \\
\hline $\begin{array}{l}\text { Waistband test } \\
\text { normal weight }\end{array}$ & 27.1 & 14.0 & 4.1 & 12.0 & 14.3 \\
$\begin{array}{l}\text { overweight } \\
\text { obese }\end{array}$ & 42.9 & 13.3 & 20.0 & 6.7 & 13.3 \\
$\quad \begin{array}{l}\text { all participants } \\
\text { Belt test }\end{array}$ & 33.3 & 41.7 & 0.0 & 16.7 & 33.3 \\
$\quad$ normal weight & 31.0 & 18.2 & 6.6 & 11.7 & 17.1 \\
$\quad$ & 12.0 & 4.0 & 8.0 & 10.0 & 4.7 \\
$\quad \begin{array}{l}\text { overweight } \\
\text { abese }\end{array}$ & 7.1 & 0.0 & 0.0 & 6.7 & 6.7 \\
$\quad$ & 41.7 & 0.0 & 0.0 & 16.7 & 16.7 \\
\hline
\end{tabular}

"Weighted means.

Note $: \mathrm{NV}=$ navel, $\mathrm{AMT}=$ anterior midline of the right thigh, $\mathrm{RS}=$ right side, $\mathrm{PMT}=$ posterior midline of the right thigh, $\mathrm{MB}=$ middle of the back.

Table 4 Mean Step Counts Recorded by Pedometer by Waistband or Belt Test at Each Placement Site by Weight Classification

\begin{tabular}{lccccr}
\hline $\begin{array}{l}\text { Weight } \\
\text { classification }\end{array}$ & NV & AMT & RS & PMT & MB \\
\hline $\begin{array}{l}\text { Waistband test } \\
\text { normal }\end{array}$ & $93.23 \pm 14.10$ & $100.70 \pm 3.42$ & $101.84 \pm 2.00$ & $100.68 \pm 3.25$ & $99.08 \pm 7.45$ \\
$\begin{array}{l}\text { overweight } \\
\text { obese }\end{array}$ & $88.14 \pm 19.13$ & $100.13 \pm 3.25$ & $102.20 \pm 2.91$ & $102.07 \pm 2.12$ & $100.87 \pm 8.27$ \\
$\begin{array}{c}\text { Belt test } \\
\text { normal }\end{array}$ & $99.78 \pm 16.77$ & $90.33^{*} \pm 18.72$ & $102.33 \pm 1.07$ & $103.17 \pm 2.73$ & $97.00 \pm 12.84$ \\
$\begin{array}{l}\text { overweight } \\
\text { obese }\end{array}$ & $99.21 \pm 2.83$ & $101.33 \pm 1.35$ & $102.00 \pm 1.65$ & $102.80 \pm 1.78$ & $101.93 \pm 4.56$ \\
\hline
\end{tabular}

*Significantly different from normal $(P<.001)$ and overweight $(P=.005)$ on the waistband test.

ANOVA analysis of mean step counts at the belt placement sites among the 3 weight groups revealed no significant differences at any of the sites: NV $(\mathrm{F}=3.85, P=.026)$, AMT $(\mathrm{F}=0.343, P=.711), \mathrm{RS}(\mathrm{F}=1.139, P=.326), \mathrm{PMT}$ $(\mathrm{F}=1.051, P=.355)$ and $\mathrm{MB}(\mathrm{F}=1.142, P=.325)$. This suggests that the belt kept the pedometer adequately positioned (vertical) for all participants at all sites. It is important to note that none of the mean step counts exceeded the $\pm 5 \%$ acceptable error rate (Table 4).

\section{Analysis of Waistband Versus Belt Test}

Paired sample $t$ tests were conducted on mean step counts for each weight classification at each placement site. No significant differences between waistband and belt 
placements were found at any site for any weight classification except at the NV site for normal participants $(P=.003)$. At the NV site, the waistband counts were significantly lower (93.23) than the belt counts (99.50, see Table 4) and exceeded the $\pm 5 \%$ acceptable error rate. Using a belt did not significantly improve accuracy except for normal-weight groups at the NV placement site.

\section{Differences in Type of Pant}

A one-way ANOVA between type of pant worn and error rate (deviation from 100 steps) on each of the waistband placement sites found no significant differences $(\mathrm{NV}: \mathrm{F}=0.036, P=.965 ;$ AMT: $\mathrm{F}=0.682, P=.509 ; \mathrm{RS}: \mathrm{F}=1.926, P=.153$; PMT: $\mathrm{F}=0.606, P=.548 ; \mathrm{MB}: \mathrm{F}=0.653, P=.523)$. The type of pant worn had no effect on error rate produced when the pedometer was worn on the waistband of the pants.

\section{Discussion}

\section{Descriptive Error Rates}

The descriptives on error rates suggest that there are differences in the accuracy of the pedometer at different waist placements. When reviewing the descriptive error rates (Table 3), there is an apparent trend for less error when using the belt compared to the waistband. It is clear that the NV position is the least desirable given the high percentages of pedometers with greater than $\pm 5 \%$ error. At every site and weight classification and on both the waistband and belt tests, the NV position had the most error. This data suggests that the NV placement site should not be recommended regardless of weight classification or placement on the waistband or belt.

For both overweight and obese participants at the NV placement site, and for obese participants at the AMT placement site, more than one third of the pedometers registered errors greater than $\pm 5 \%$ on the waistband test. The AMT site is the placement most often recommended by pedometer manufacturers, yet for obese participants, it produced a $41.7 \%$ error rate. This is an issue to consider, because when obese participants participate in physical activity and monitor it with a pedometer that doesn't read accurately (ie, too few steps), they may falsely assume they are not sufficiently active. Having spuriously low readings may cause frustration and withdrawal from the activity. When the pedometers were placed at the RS site, a reduction in error rates was observed (Table 3).

When using a belt compared to the waistband, the AMT placement improved for obese participants (from $41.7 \%$ to $0.0 \%$ ). The RS placement registered no errors for overweight and obese participants when wearing a belt. These descriptive data show a trend for improved accuracy when wearing a belt. It may be that the belt secures the pedometer more tightly to the body of participants with excessive abdominal fat, keeping it in the vertical position.

\section{Differences in Placement Sites}

Significance tests revealed that weight classification had no influence on pedometer accuracy at the NV, RS, PMT, and MB placements during the waistband 
test. Even though there were no significant differences between weight groups on these 4 placements, it does not mean that the pedometers were all accurate. At the NV placement site, all pedometers registered fewer that 95 steps as did the pedometers of the obese group at the AMT site (Table 4). Even though there were no significant differences, lower step counts at the NV placement site for all weight groups and at the AMT placement site for obese participants indicate that these are not appropriate placements sites for obese participants. Table 4 reveals that the RS, PMT, and MB placement sites produce the most accurate step counts regardless of weight group. These 3 placement sites (where all mean step counts are within acceptable limits) can be used for any weight group. However, only the RS placement permits the user to read the step count during activity without having to remove the pedometer from the waistband.

Statistical tests among weight groups on the belt test found no significant step count differences at any of the 5 placement sites with all of the values falling within the acceptable $\pm 5 \%$ error rate. When using a belt, all pedometers in all weight classifications performed properly.

\section{Waistband Versus Belt Test}

It was anticipated that wearing a belt would improve accuracy over wearing the pedometer directly on the waistband. Results of the paired $t$ tests on pedometerrecorded steps between waistband and belt placement sites by weight group indicated no significant differences except for normal participants at the NV site. At the NV placement site, normal participants had fewer steps (Table 4) when the pedometer was on the waistband $(P=.003)$.

\section{Type of Pant Worn}

Participants in this study wore a Docker-type pant, Levi-type jeans, or sweat pants when they participated. This information was gathered to determine if the type of pant worn (ie, differences in waistband stability) had an effect on pedometer accuracy. ANOVA analysis found no significant differences between the type of pant worn for each of the waistband placement sites. It was concluded that the type of pant the participants wore had no effect on error rate. Further research is needed in this area to confirm this result.

\section{Comparisons With Other Research}

When using a spring-lever pedometer, it is important to maintain a vertical position in order to obtain accurate step counts. This issue was evaluated in a study by Crouter et al. ${ }^{8}$ in which a spring-lever pedometer was compared to a piezoelectric pedometer. One of the main findings indicated that spring-lever pedometers were more influenced by pedometer tilt. The piezoelectric pedometer was not influenced by tilt. In the current study, statistical comparisons using paired $t$ tests did not show significant differences between the waistband and belt tests except for normal participants at the NV site. However, the descriptive data on error rates demonstrated a trend for less error when a belt was worn. More research needs to be conducted on the usefulness of a belt when wearing spring-lever pedometers. 
Jago et al. ${ }^{14}$ evaluated pedometer accuracy in boys ages 10 to 15 years at different locations (right hip, center (navel), and left hip) while wearing an elastic belt. They found that pedometer location did not make a difference in accuracy. The differences between the methods of Jago et al. and those of the current study are that in the current study, 5 placement sites were measured and both waistband and belt placements were evaluated. Jago et al. evaluated the pedometer on the right and left hips, which are generally accepted as being similar placement sites; therefore, essentially only 2 sites (navel and side) were considered, and all participants anchored the pedometer to a belt. The current study evaluated 5 different locations all on the right side of the body with and without a belt. Jago et al. found that adiposity did not make a difference in accuracy. The current study confirms the results of Jago et al. for the belt but found significant differences when using the waistband of pants.

Two additional studies were conducted on adults and produced mixed results regarding pedometer error related to body weight and placement of the pedometer. Swartz et al. ${ }^{13}$ studied 3 pedometer placements (midline of the thigh front, midaxillary line, and posterior midline of thigh) and found that waist circumference and BMI did not affect the accuracy of the pedometer (Yamax SW-200) at any of the placement sites. In contrast, Shepherd et al. ${ }^{11}$ found that when the pedometer (Sportline) was placed on the side, more error resulted with obese individuals than with normal-weight individuals when participating in 4 different activities. These studies are not directly comparable to the current study because of differences in the age of participants, type of pedometer, and variation in placement sites.

\section{Strengths and Limitations}

The major strength of this study was the testing of 5 different pedometer sites and the use of normal, overweight, and obese participants. Manufacturers generally recommend 1 placement site and most often do not justify that recommendation and do not provide instructions related to weight classification. Additionally, the pedometers in the current study were evaluated with a shake test prior to and after use in this study to ensure they were accurate.

There are several limitations to this study that should be considered. Only 1 pedometer brand (Walk4Life LS 2505) was used. The results of this study apply specifically to this pedometer brand. More research is needed on other spring-lever and piezoelectric pedometers to determine the generalizability of these results. Testing of other spring-lever pedometers would be useful to determine if current findings are typical of all spring-lever pedometers. Results of this study are limited to youth ages 10 to 12 years. Further research is needed on older youth and adults to confirm these findings and make comparisons across ages.

\section{Conclusions}

The RS, PMT, and MB placement sites produced the most accurate pedometer step counts on waistband tests for all weight classifications. Of these 3 sites, the RS placement is recommended because of the ease of reading the pedometer during activity. With additional research on additional pedometer brands, manufacturers 
may consider changing their recommendation for pedometer placement to the right side. The belt did not produce statistically significant improvement when compared to the waistband except at the NV site for normal weight participants. However, descriptive statistics of individual pedometer error rates at the various placement sites for each weight classification suggested a trend in favor of the belt. The type of pant worn had no effect on error rate when the pedometer was worn on the waistband of the pants.

\section{References}

1. Schneider PL, Crouter SE, Bassett DR. Pedometer measures of free-living physical activity: comparison of 13 models. Med Sci Sports Exerc. 2004;36(2):331-335.

2. Crouter SE, Schneider PL, Karabulut M, Bassett DR. Validity of 10 electronic pedometers for measuring steps, distance, and energy costs. Med Sci Sports Exerc. 2003;35(8):1455-1460.

3. Beets MW, Patton MM, Edwards S. The accuracy of pedometer steps and time during walking in children. Med Sci Sports Exerc. 2005;37(3):513-520.

4. Bassett DR, Strath SJ. Use of pedometers to assess physical activity. In: Welk GJ, ed. Physical Activity Assessments for Health-Related Research. Champaign, Ill: Human Kinetics; 2002:163-177.

5. Beighle A, Pangrazi RP, Vincent SD. Pedometers, physical activity, and accountability. Phys Educ, Rec, Dance. 2001;72(9):16-19.

6. Le Masurier GC, Beighle A, Corbin CB, et al. Pedometer-determined physical activity levels of youth. J Phys Act Health. 2005;2:159-168.

7. Vincent SD, Pangrazi RP, Raustorp A, Tomson LM, Cuddihy TF. Activity levels and body mass index of children in the United States, Sweden, and Australia. Med Sci Sports Exerc. 2003;35:1367-1373.

8. Crouter SE, Schneider PL, Bassett DR. Spring-levered versus piezo-electric pedometer accuracy in overweight and obese adults. Med Sci Sports Exerc. 2005;37:1673-1679.

9. Schneider PL, Crouter SE, Lukajic O, Bassett DR. Accuracy and reliability of 10 pedometers for measuring steps over a 400-m walk. Med Sci Sports Exerc. 2003;35(10):17791784 .

10. Bassett DRJ, Ainsworth BE, Leggett SR, et al. Accuracy of five electronic pedometers for measuring distance walked. Med Sci Sports Exerc. 1996;28(8):1071-1077.

11. Shepherd EF, Toloza E, McClung CD, Schmalzried TP. Step activity monitor: increased accuracy in quantifying ambulatory activity. J Orthop Res. 1999;17:703-708.

12. Tudor-Locke CE, Myers AM. Methodological considerations for researchers and practitioners using pedometers to measure physical (ambulatory) activity. Res $Q$ Exerc Sport. 2001;72:1-12.

13. Swartz AM, Bassett DR, Moore JB, Thompson DL, Strath SJ. Effects of body mass index on the accuracy of an electronic pedometer. Int J Sports Med. 2003;24:588-592.

14. Jago R, Watson K, Baranowski T, et al. Pedometer reliability, validity and daily activity targets among 10- to 15-year-old boys. J Sports Sci. 2006;24:241-251.

15. Bassett DR, Ainsworth BE, Swartz AM, Strath SJ, O'Brien WL, King GA. Validity of four motions sensors in measuring moderate intensity physical activity. Med Sci Sports Exerc. 2000;32(9, suppl):S471-S480.

16. Vincent SD, Sidman CL. Determining measurement error in digital pedometers. Meas Phys Educ Exerc Sci. 2003;7(1):19-24.

17. Lohman TG, Roche AF, Martorell R, eds. Anthropometric Standardization Reference Manual. Champaign, Ill: Human Kinetics; 1988.

18. Cole TJ, Bellizzi MC, Flegal KM, Dietz WH. Establishing a standard definition for child overweight and obesity worldwide: international survey. BMJ. 2000;320:1240-1245. 